\title{
Gene Therapy in a Large Animal Model of PDE6A-Retinitis Pigmentosa
}

\section{OPEN ACCESS}

Edited by: Mark P. Burns,

Georgetown University, United States

Reviewed by:

Tiansen $\mathrm{Li}$,

National Institutes of Health

United States

Breandan Kennedy,

University College Dublin, Ireland

${ }^{*}$ Correspondence:

Freya M. Mowat

fmmowat@ncsu.edu

Simon M. Petersen-Jones

peter315@cvm.msu.edu

Specialty section:

This article was submitted to

Neurodegeneration,

a section of the journal

Frontiers in Neuroscience

Received: 06 February 2017

Accepted: 01 June 2017

Published: 20 June 2017

Citation:

Mowat FM, Occelli LM, Bartoe JT, Gervais KJ, Bruewer AR, Querubin J,

Dinculescu A, Boye SL,

Hauswirth WW and

Petersen-Jones SM (2017) Gene Therapy in a Large Animal Model of

PDE6A-Retinitis Pigmentosa.

Front. Neurosci. 11:342.

doi: 10.3389/fnins.2017.00342

\section{Freya M. Mowat ${ }^{1,2 *}$, Laurence M. Occelli ${ }^{1}$, Joshua T. Bartoe ${ }^{1}$, Kristen J. Gervais ${ }^{1}$, Ashlee R. Bruewer ${ }^{1}$, Janice Querubin ${ }^{1}$, Astra Dinculescu ${ }^{3}$, Sanford L. Boye ${ }^{3}$, William W. Hauswirth ${ }^{3}$ and Simon M. Petersen-Jones ${ }^{1 *}$}

'Department of Small Animal Clinical Sciences, College of Veterinary Medicine, Michigan State University, East Lansing, MI, United States, ${ }^{2}$ Department of Clinical Sciences, College of Veterinary Medicine, North Carolina State University, Raleigh, NC, United States, ${ }^{3}$ Department of Ophthalmology, University of Florida College of Medicine, Gainesville, FL, United States

Despite mutations in the rod phosphodiesterase 6-alpha (PDE6A) gene being well-recognized as a cause of human retinitis pigmentosa, no definitive treatments have been developed to treat this blinding disease. We performed a trial of retinal gene augmentation in the Pde6a mutant dog using Pde6a delivery by capsid-mutant adeno-associated virus serotype 8, previously shown to have a rapid onset of transgene expression in the canine retina. Subretinal injections were performed in 10 dogs at 29-44 days of age, and electroretinography and vision testing were performed to assess functional outcome. Retinal structure was assessed using color fundus photography, spectral domain optical coherence tomography, and histology. Immunohistochemistry was performed to examine transgene expression and expression of other retinal genes. Treatment resulted in improvement in dim light vision and evidence of rod function on electroretinographic examination. Photoreceptor layer thickness in the treated area was preserved compared with the contralateral control vector treated or uninjected eye. Improved rod and cone photoreceptor survival, rhodopsin localization, cyclic GMP levels and bipolar cell dendrite distribution was observed in treated areas. Some adverse effects including foci of retinal separation, foci of retinal degeneration and rosette formation were identified in both AAV-Pde6a and control vector injected regions. This is the first description of successful gene augmentation for Pde6a retinitis pigmentosa in a large animal model. Further studies will be necessary to optimize visual outcomes and minimize complications before translation to human studies.

Keywords: retinitis pigmentosa, Pde6 mutation, gene therapy, canine model, adeno-associated virus

\section{INTRODUCTION}

Retinitis pigmentosa (RP) is a major cause of heritable vision loss affecting $\sim 1$ in 4,000 people (Hartong et al., 2006). It is a genetically heterogenous group of conditions; at the time of writing 67 mapped genes and loci for non-syndromic RP were listed on the RetNet website (the Retinal Information Network https://sph.uth.edu/retnet/home.htm). In many forms of RP the mutated genes are specific to rod photoreceptors leading to rod degeneration and depending on the function of the gene product, a period of abnormal, or absent, rod function may precede rod death. Although 
cone photoreceptors in many forms of RP do not express the mutant gene, cone degeneration will occur as a consequence of rod degeneration. Initial symptoms of RP result from reduced, or absence of rod function resulting in night blindness. With progression an impairment of cone mediated vision develops leading to visual field constriction and in many cases, complete blindness. Mutations of the alpha or beta subunits of the rod phosphodiesterase (PDE6) result in autosomal recessive RP (arRP). PDE6-alpha (PDE6A; OMIM 180071) and PDE6-beta (PDE6B; OMIM 180072) mutations each account for $\sim 4 \%$ of cases of arRP (McLaughlin et al., 1995; Dryja et al., 1999).

The PDE6 complex in rod photoreceptors is composed of two active subunits, alpha and beta, and a pair of gamma inhibitory subunits. Removal of the gamma inhibitory subunits during phototransduction by the activity of transducin, allows the alpha and beta dimer to hydrolyze many cyclic GMP (cGMP) molecules, causing gated channels to close and plasma membrane hyperpolarization to occur (Farber, 1995). Animals lacking functional alpha or beta PDE6 subunit show a reduction in the phototransductioninduced hydrolysis of cGMP resulting in elevated cGMP levels, unregulated influx of calcium, primary rod cell death, and subsequent secondary cone loss (Wensel et al., 2016).

A variety of mouse models for $P D E 6 B$ arRP have been widely used to study disease mechanisms and treatment, including $P d e 6 b^{r d 1 / r d 1}$ which has a null mutation, and peak rod loss at day 14, (Keeler, 1966; Pittler et al., 1993; Hackam et al., 2004) and the milder $P d e 6 b^{r d 10 / r d 10}$ missense mutation in which rod loss peaks at day 25 (Gargini et al., 2007; Barhoum et al., 2008). The $P d e 6 b^{r d 10 / r d 10}$ mouse retina initially contains low levels of Pde6b and retains a rod-mediated electroretinogram response prior to degeneration (Chang et al., 2007). Additional mutagenesis models have been described for Pde6b in the mouse, exhibiting a variety of severities of retinal disease (Hart et al., 2005; Chang et al., 2007). Irish setter dogs with a null mutation in $P d e 6 b$ provide a large animal model for PDE6B arRP (Farber et al., 1992; Suber et al., 1993). Affected dogs lack Pde6b activity and have elevated retinal cGMP levels arresting normal photoreceptor development from day 13 , resulting in retinal degeneration beginning at day 25; rod loss is followed by a progressive loss of cones leading to blindness (Aguirre et al., 1978; Farber et al., 1992). Successful gene therapy studies were initially described for the milder phenotypes of the $P d e 6 b^{r d 10 / r d 10}$ mouse (Pang et al., 2008, 2011) and Pde6b ${ }^{H 620 Q}$ hypomorphic mouse model (Davis et al., 2008; Tosi et al., 2011). The early onset, rapid degeneration of the $P d e 6 b^{r d 1 / r d 1}$ mouse proved to be more challenging to treat with gene therapy, with only partial rescue of the phenotype described (Bennett et al., 1996; Jomary et al., 1997; Kumar-Singh and Farber, 1998; Takahashi et al., 1999; Guo et al., 2015). A recent description of an additional mutation in Gpr179 in the Pde6b $b^{r d 1 / r d 1}$ mouse line may explain some of the difficulty in rescuing the $P d e 6 b^{r d 1 / r d 1}$ phenotype (Nishiguchi et al., 2015). Following breeding to remove the co-inherited Gpr179 mutation, gene therapy rescued the Pde6b $b^{r d 1 / r d 1}$ phenotype. Successful and sustained rescue of the $P d e 6 b$ mutant dog phenotype with adeno-associated viral vectors encoding canine $P d e 6 b$ when administered by subretinal injection at 20 days of age has been described (Petit et al., 2012; Pichard et al., 2016).

More recently, several mouse models for PDE6A arRP have been developed. The severity and rapidity of retinal degeneration of each model is dependent on the gene mutation, varying from slow to rapid (Sakamoto et al., 2009; Sothilingam et al., 2015). We previously described a recessively inherited Pde6a null mutation (one base-pair deletion at codon 616 resulting in a frameshift and premature stop codon) in the Cardigan Welsh Corgi breed of dog causing an early-onset and rapid rod and subsequent cone degeneration (Petersen-Jones et al., 1999; Tuntivanich et al., 2009). Outer nuclear layer cell death was present from post-natal day 21 and peaked at post-natal day 27. Cell loss is not related to caspase 3 activity in mouse (Sothilingam et al., 2015) and dog (Tuntivanich et al., 2009) models, indicating a non-caspase dependent mechanism of cell death. Pde6a mouse models have reduced amounts of retinal Pde6b in parallel with the decrease in Pde6a (Sakamoto et al., 2009) and the retina of $P d e 6 a$ mutant dogs also lacks beta and gamma subunits of Pde6 indicating a requirement for Pde6a for the normal production or maintenance of the other subunits in the rod outer segment (Petersen-Jones et al., 1999; Tuntivanich et al., 2009). This is in contrast to animal models with Pde6b null mutations in which expression of the other Pde6 subunits is retained (Pittler and Baehr, 1991; Suber et al., 1993). Therapeutic trials using the Pde6a ${ }^{n m f 363}$ mouse model have been reported (Wert et al., 2013, 2014). This particular mouse model has one of the less severe phenotypes. Therapy in this model was successful when performed both prior to photoreceptor loss (Wert et al., 2013) but also when $\sim 50 \%$ of photoreceptors had degenerated (Wert et al., 2014). Our initial attempts to rescue the Pde6a mutant dog with AAV serotype 5 gene augmentation therapy were unsuccessful (Petersen-Jones, unpublished findings). We hypothesized that because of the severity of the model, transgene expression would be required at a very early age, requiring delivery of treatment to the retina at a stage at which it is still developing and that we would need highly efficient, rapid-onset, viral vectors. A high efficiency adeno-associated virus serotype8 with capsid mutation was successful in rescuing the rapid degeneration seen in the Aipl1 mutant mouse (Ku et al., 2015) and the Pde6a $a^{n m f 363}$ mouse model, (Wert et al., 2013) even when delivered at more advanced stages of disease (Wert et al., 2014). We have shown that tyrosine capsid-mutant AAV8 viral vectors have an onset of gene expression within 2-3 days in the canine retina, (Mowat et al., 2014) and therefore show promise for the rescue of the rapid degeneration seen in the Pde6a mutant dog.

Currently there are no definitive treatments for RP associated with retinal PDE6 mutations. Recent phase I/II clinical trials have demonstrated the safety and efficacy of gene augmentation using adeno-associated viral vectors in patients with Leber Congenital Amaurosis due to RPE65 gene mutations (Bainbridge et al., 2008; Cideciyan et al., 2008, 2009a,b; Hauswirth et al., 2008; Maguire et al., 2008, 2009; Simonelli et al., 2010). Experimental efficacy in Pde6b models will allow translation into human clinical trials in the near future, however only 
limited preclinical trials have been performed in PDE6A models.

In this publication, we present the results of tyrosine capsidmutant AAV8 Pde6a gene therapy delivered by subretinal injection to young Pde6a mutant dogs. We show that treatment results in partial return of visual function as determined by electroretinography and objective vision assessment. The treatment effect is sustained for at least 4 months, and preserves photoreceptors in the treated area. Treatment reverses retinal cGMP accumulation, reduces mislocalization of rhodopsin, limits glial activation, and maintains dendritic arbors of rod bipolar cells. However, within the treated areas adverse treatment related effects were seen. These include persistent regions of retinal separation and rosette formation.

\section{MATERIALS AND METHODS}

\section{Subjects}

Animals were bred and housed in a facility at Michigan State University. Animals $(n=10)$ were all homozygous for a mutation in Pde6a (Petersen-Jones et al., 1999; Tuntivanich et al., 2009). Animals were housed on a 12-h light: dark cycle, and care was in compliance with the ARVO Statement for the Use of Animals in Ophthalmic and Visual Research, with all procedures being performed with approval from the Michigan State University Institutional Animal Care and Use Committee.

\section{AAV Constructs}

Recombinant AAV vectors were manufactured and purified by previously described methods, (Hauswirth et al., 2000; Zolotukhin et al., 2002; Zhong et al., 2008; Pang et al., 2011; Petrs-Silva et al., 2011) including purification and concentration by column chromatography. Vector titer was determined by realtime PCR and aliquots were resuspended in balanced salt solution (BSS, Alcon Laboratories, Fort Worth, TX, USA) containing 0.014\% tween 20 (Sigma Aldrich, St Louis MO). The vector used was an AAV8 capsid with a single tyrosine to phenylalanine mutation at position 733 (Petrs-Silva et al., 2011) containing a construct expressing either canine Pde6a cDNA (AAV-Pde6a) or humanized green fluorescent protein cDNA $(A A V-G F P)$ driven by the ubiquitous truncated chimeric CMV-chicken beta-actin promoter (smCBA).

\section{Subretinal Injections}

All vectors were diluted to $4.19 \times 10^{11}$ viral genomes $/ \mathrm{ml}$ using sterile balanced salt solution (BSS) before injection. Under general anesthesia, 50-100 $\mu$ l of vector preparation was delivered by subretinal injection as previously described (Petersen-Jones et al., 2009; Mowat et al., 2014). Injections were placed in the superior tapetal portion of the retina, close to the nasal, or temporal aspect of the optic nerve head. Immediately after subretinal injection, all dogs received a subconjunctival injection of $2 \mathrm{mg}$ dexamethasone solution and $2 \mathrm{mg}$ of methylprednisolone acetate. Dogs were injected at a mean of 36 days of age (range 2944 days, Table 1). One eye from each dog was injected with $A A V$ $P d e 6 a$, the contralateral eye was uninjected $(n=5)$ or injected with $A A V-G F P(n=5)$.
TABLE 1 | Details of dogs used for experimental procedures.

\begin{tabular}{|c|c|c|c|c|c|}
\hline $\begin{array}{l}\text { Animal } \\
\text { number }\end{array}$ & $\begin{array}{l}\text { Age at } \\
\text { injection } \\
\text { (days) }\end{array}$ & $\begin{array}{l}\text { Injection } \\
\text { OD }\end{array}$ & $\begin{array}{l}\text { Injection } \\
\text { OS }\end{array}$ & $\begin{array}{l}\text { In vivo } \\
\text { data } \\
\text { collection }\end{array}$ & $\begin{array}{c}\text { Age at } \\
\text { euthanasia } \\
\text { (days) }\end{array}$ \\
\hline $10-023$ & 30 & $\begin{array}{l}\text { AAV2/8mut } \\
\text { 733.CBAp. } \\
\text { cPDE6a }\end{array}$ & None & $\begin{array}{l}\text { ERG } \\
\text { VT: ob } \\
\text { FI }\end{array}$ & 92 \\
\hline $11-001$ & 29 & $\begin{array}{l}\text { AAV2/8mut } \\
\text { 733.CBAp. } \\
\text { cPDE6a }\end{array}$ & None & $\begin{array}{l}\text { ERG } \\
\text { VT: ob } \\
\text { FI }\end{array}$ & 125 \\
\hline $11-006$ & 29 & $\begin{array}{l}\text { AAV2/8mut } \\
\text { 733.CBAp. } \\
\text { cPDE6a }\end{array}$ & None & $\begin{array}{l}\text { ERG } \\
\text { VT: ob } \\
\text { FI }\end{array}$ & 125 \\
\hline $11-007$ & 29 & $\begin{array}{l}\text { AAV2/8mut } \\
\text { 733.CBAp. } \\
\text { cPDE6a }\end{array}$ & None & $\begin{array}{l}\text { ERG } \\
\text { VT: ob } \\
\mathrm{FI}\end{array}$ & 125 \\
\hline $11-008$ & 29 & $\begin{array}{l}\text { AAV2/8mut } \\
\text { 733.CBAp. } \\
\text { cPDE6a }\end{array}$ & None & $\begin{array}{l}\text { ERG } \\
\text { VT: ob } \\
\text { FI }\end{array}$ & 125 \\
\hline $12-046$ & 42 & $\begin{array}{l}\text { AAV2/8mut } \\
\text { 733.CBAp. } \\
\text { cPDE6a }\end{array}$ & $\begin{array}{l}\text { AAV2/8mut } \\
\text { 733.CBAp. } \\
\text { GFP }\end{array}$ & $\begin{array}{l}\text { ERG } \\
\text { VT: ob/cd } \\
\text { OCT } \\
\text { FI }\end{array}$ & 208 \\
\hline $12-047$ & 41 & $\begin{array}{l}\text { AAV2/8mut } \\
\text { 733.CBAp. } \\
\text { cPDE6a }\end{array}$ & $\begin{array}{l}\text { AAV2/8mut } \\
\text { 733.CBAp. } \\
\text { GFP }\end{array}$ & $\begin{array}{l}\text { ERG } \\
\text { VT: ob/cd } \\
\text { OCT } \\
\text { FI }\end{array}$ & 208 \\
\hline $12-048$ & 41 & $\begin{array}{l}\text { AAV2/8mut } \\
\text { 733.CBAp. } \\
\text { cPDE6a }\end{array}$ & $\begin{array}{l}\text { AAV2/8mut } \\
\text { 733.CBAp. } \\
\text { GFP }\end{array}$ & $\begin{array}{l}\text { ERG } \\
\text { VT: ob/cd } \\
\text { OCT } \\
\text { FI }\end{array}$ & 208 \\
\hline $12-060$ & 44 & $\begin{array}{l}\text { AAV2/8mut } \\
\text { 733.CBAp. } \\
\text { cPDE6a }\end{array}$ & $\begin{array}{l}\text { AAV2/8mut } \\
\text { 733.CBAp. } \\
\text { GFP }\end{array}$ & $\mathrm{FI}$ & 58 \\
\hline $12-061$ & 44 & $\begin{array}{l}\text { AAV2/8mut } \\
\text { 733.CBAp. } \\
\text { cPDE6a }\end{array}$ & $\begin{array}{l}\text { AAV2/8mut } \\
\text { 733.CBAp. } \\
\text { GFP }\end{array}$ & ERG, FI & 72 \\
\hline
\end{tabular}

$O D$, Right eye; OS, left eye; ERG, electroretinography; VT, vision testing; ob, obstacle course; cd, 4 tunnel choice device; Fl, fundus imaging; OCT, optical coherence tomography imaging.

\section{Ophthalmic Evaluation and Fundic Imaging}

Slit-lamp biomicroscopy (SL15, Kowa, Japan), binocular indirect ophthalmoscopy (Welch Allyn, Skaneateles Falls, NY, USA) and wide-angle color and fluorescent digital fundus imaging (RetCam II, Clarity Medical Systems, Pleasanton CA) were performed immediately following injection and then at regular intervals. 


\section{Confocal Scanning Laser Ophthalmoscopy and Spectral Domain Optical Coherence Tomography}

Confocal scanning laser ophthalmoscopy (cSLO) and spectral domain optical coherence tomography (SD-OCT) imaging (Spectralis HRA+OCT, Heidelberg Engineering, Carlsbad CA USA) was performed under general anesthesia. Pupils were dilated with tropicamide (Tropicamide Ophthalmic Solution UPS 1\%; Falcon Pharmaceuticals Ltd., Fort Worth, TX, USA), and the eyes were maintained in primary gaze for imaging. Wide-field fundus images were obtained by cSLO using infrared imaging ( $820 \mathrm{~nm}$ laser) and fluorescent imaging (488 $\mathrm{nm}$ laser). Retinal cross-section images were obtained using SD-OCT with an $820 \mathrm{~nm}$ wavelength laser and a $30^{\circ}$ lens. The injected area was imaged in both the $A A V-P d e 6 a$ treated and $A A V$-GFP treated eye $(n=3$ dogs). Images were obtained at 1,3 , and 5 months following injection. Quantification of OCT segment thickness was performed using the integrated Heidelberg Eye Explorer (Heyex) software. The thickness of the outer nuclear layer was measured in six regions throughout the injected area, and a mean value was calculated per eye, per timepoint to compare statistically.

\section{Electroretinography}

Bilateral electroretinography (ERG) was performed at $0.5,1$, 3 , and 4 months following injection, using methods previously described (Annear et al., 2011). The amplitude of the peak scotopic threshold response (STR; detected at a flash luminance below that needed to elicit a b-wave) at each timepoint was calculated and used in analysis.

\section{Vision Testing}

Subjective assessment of visual ability was used in a subset of animals (see Table 1). This was performed using an obstacle course, through which the animals navigated using either the $A A V-P d e 6 a$ treated eye or the control eye. Objective assessment of a subset of animals was performed using a previously described four-choice device, (Gearhart et al., 2008) allowing quantifiable testing of visual ability at a range of light intensities. Light intensities were adjusted to range from a dim light that tests rod only vision through mesopic to photopic levels (full room light).

\section{Tissue Processing and Sectioning}

Animals were euthanized using barbiturate overdose (Fatal Plus, Vortech Pharmaceuticals, Dearborn, MI, USA) 0.5-6 months following injection (Table 1). Eyes were prepared for sectioning as previously described (Mowat et al., 2013, 2014). Globes were sectioned sagitally through the vertical meridian. Fourteen micron frozen sections were taken from the area including the optic nerve and of the nasal and temporal retina, to encompass both injected and uninjected areas within the same eye.

\section{Immunohistochemistry}

Reagents utilized, and basic immunohistochemistry (IHC) protocols performed were as previously described (Mowat et al., 2014). Specific antibodies and conditions utilized in this study are outlined in Table 2.

\section{Imaging and Quantification of Immunolabeled Retinal Sections}

Slides were imaged using a fluorescence microscope (Nikon Eclipse 80i, Nikon instruments Inc., Melville, NY, USA) using commercial image capture software (MetaVue, Molecular Devices, Sunnydale, CA, USA). For rod and cone counts, slides co-labeled for Pde6 and cones (with human cone arrestin antibody) were imaged. Within each section, three images were taken from the superior area (in injected eyes, defined by positive PDE6 IHC or GFP expression). In control eyes, the region imaged was defined by the site of injection of the AAV-Pde6a treated eye. Three sections were imaged, giving 9 regions per eye. A mean value was calculated for cone and rod number in a $500 \mu \mathrm{m}$ section of retina was calculated as previously described (Mowat et al., 2013). Representative images of immunohistochemistry were also captured using a confocal microscope (Olympus FluoView 1000, Center Valley, CA).

\section{Statistical Analysis}

Statistical analysis was performed using GraphPad Prism 5.0a (GraphPad Software Inc, La Jolla, CA, USA). For vision testing data, a two-way analysis of variance was used to assess the effect

TABLE 2 | Primary antibodies used for immunohistochemistry.

\begin{tabular}{llll}
\hline Antigen target & $\begin{array}{l}\text { Antibody details and } \\
\text { working dilution }\end{array}$ & Manufacturer & $\begin{array}{l}\text { Secondary antibody and } \\
\text { concentration (all from Invitrogen } \\
\text { Corp. Carlsbad CA, USA) }\end{array}$ \\
\hline PDE6 (all isoforms) & Rabbit polyclonal 1:5000 & Cytosignal, Irvine, CA, USA & AlexaFluor anti-rabbit 546 1:500 \\
PDE6B & Rabbit polyclonal 1:250 & Novus Biologicals, Littleton, CO, USA & AlexaFluor anti-rabbit 546 1:500 \\
Rhodopsin & Mouse monoclonal 1:10 & Labvision (Thermoscientific), Kalamazoo Ml, USA & AlexaFluor anti-mouse 488 1:500 \\
$\begin{array}{l}\text { Cone arrestin (LUMif) } \\
\text { (Li et al., 2003) }\end{array}$ & Rabbit polyclonal 1:10,000 & Kind gift of Cheryl M. Craft, University of Southern & AlexaFluor anti-rabbit 546 1:500 \\
$\begin{array}{l}\text { Protein kinase-C alpha } \\
\text { Glial Fibrillary Acidic Protein }\end{array}$ & Mouse monoclonal 1:500 & California & BD Biosciences, San Diego, CA, USA \\
Cyclic GMP & Rabbit polyclonal 1:500 & Dako North America, Carpinteria, CA, USA & AlexaFluor anti-mouse 488 1:500 \\
& Sheep formaldehyde & Gift of Dr. HWM Steinbusch, Maastricht University & AlexaFluor anti-sheep 488, 1:500
\end{tabular}


of treatment and time to exit the device or light intensity on the variable examined. A Bonferroni post-test correction was used to compare replicate means. For rod and cone counts comparing treated eyes with contralateral untreated or GFP treated eyes, a two-tailed paired student's $t$-test was performed. Effects were considered significant if $P<0.05$. In all figures, graphs represent mean \pm standard error of the mean.

\section{RESULTS}

\section{Pde6a Gene Augmentation Results in a Scotopic Threshold Response in the ERG and Improved Dim Light Vision}

Untreated and control AAV8-GFP injected eyes of Pde6a mutant dogs did not have detectable rod ERG responses. There were no scotopic threshold responses, and the dark- and light-adapted bwave thresholds were similar and apart from the lowest stimulus intensities the b-wave amplitudes were similar in amplitude (Figure 1A). At 1 month following subretinal injection with $A A V$-Pde6a evidence of rod-mediated ERG responses were recordable in 5 out of the 9 dogs (one dog was terminated prior to the 1 month ERG). In each of the five dogs with a detectable ERG difference the main effect of therapy was the introduction of a scotopic threshold response (STR) which is a rod driven response (Figures 1A-C) (Sieving et al., 1986). The STR response was sustained to 4 months after treatment (Figure 1D). The same dogs had a dark-adapted ERG b-wave in response to flashes just above threshold with a longer implicit time than in controls. The longer implicit time is more in

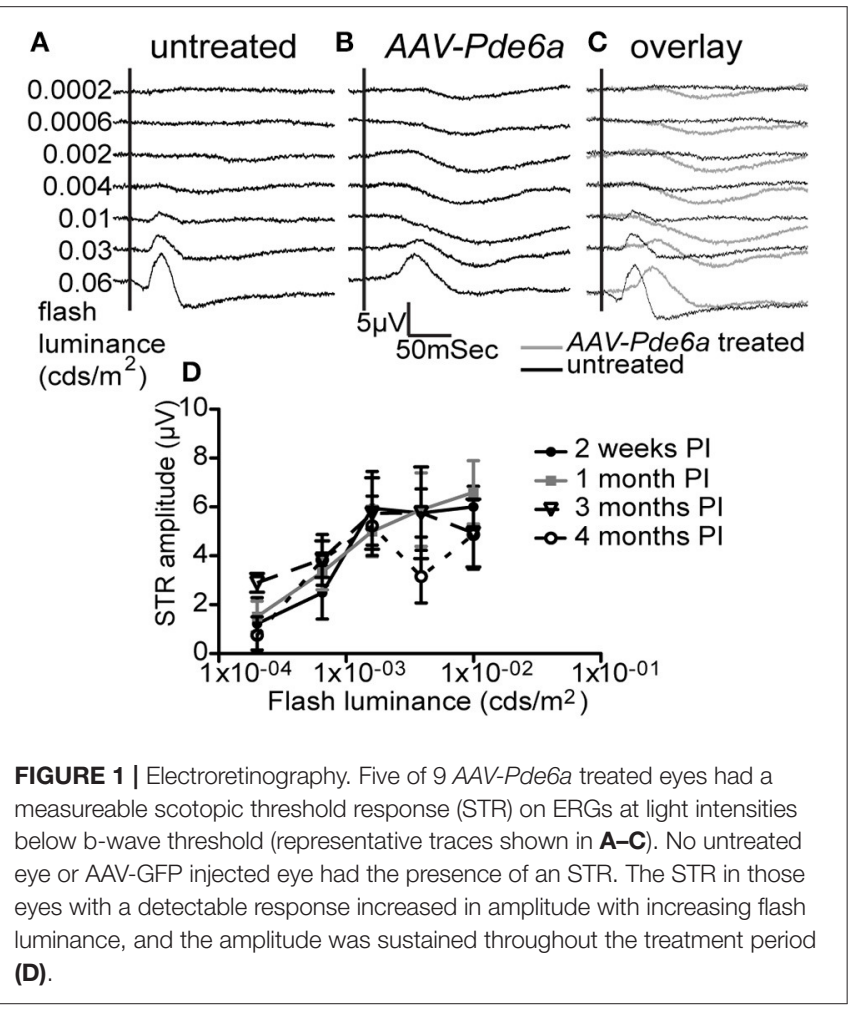

keeping with a rod rather than cone response, although the darkadapted b-wave threshold and amplitude was not improved by therapy.

Dogs with recordable STR responses also showed an improvement in dim light visual function in the $A A V-P d e 6 a$ treated eye, confirming the ERG findings of improved rod function (Figure 2 and Supplementary Video 1). At a low light intensity $\left(0.02 \mathrm{~cd} / \mathrm{m}^{2}\right)$, animals tended to navigate more rapidly through the device using the $A A V-P d e 6 a$ treated eye (Figure 2A) and made fewer incorrect first choice of exit tunnel (Figure 2B). Overall eyes treated with the therapeutic vector (AAV-Pde6a) had significantly improved visual function compared to the control eyes (AAV-GFP) ( $p<0.01$ ANOVA), although statistical significance was only achieved at certain timepoints following injection. To further investigate rod-mediated vision, at 5 months following injection, a range of extremely low light levels were tested (range $0.02-0.003 \mathrm{~cd} / \mathrm{m}^{2}$ ), and animals navigated significantly more quickly (Figure 2C) and with fewer mistakes (Figure 2D) using AAV-Pde6a treated eyes compared with $A A V$-GFP treated eyes. Brighter light (cone-mediated) vision was maintained in both $A A V$-Pde6a and $A A V$-GFP treated eyes.

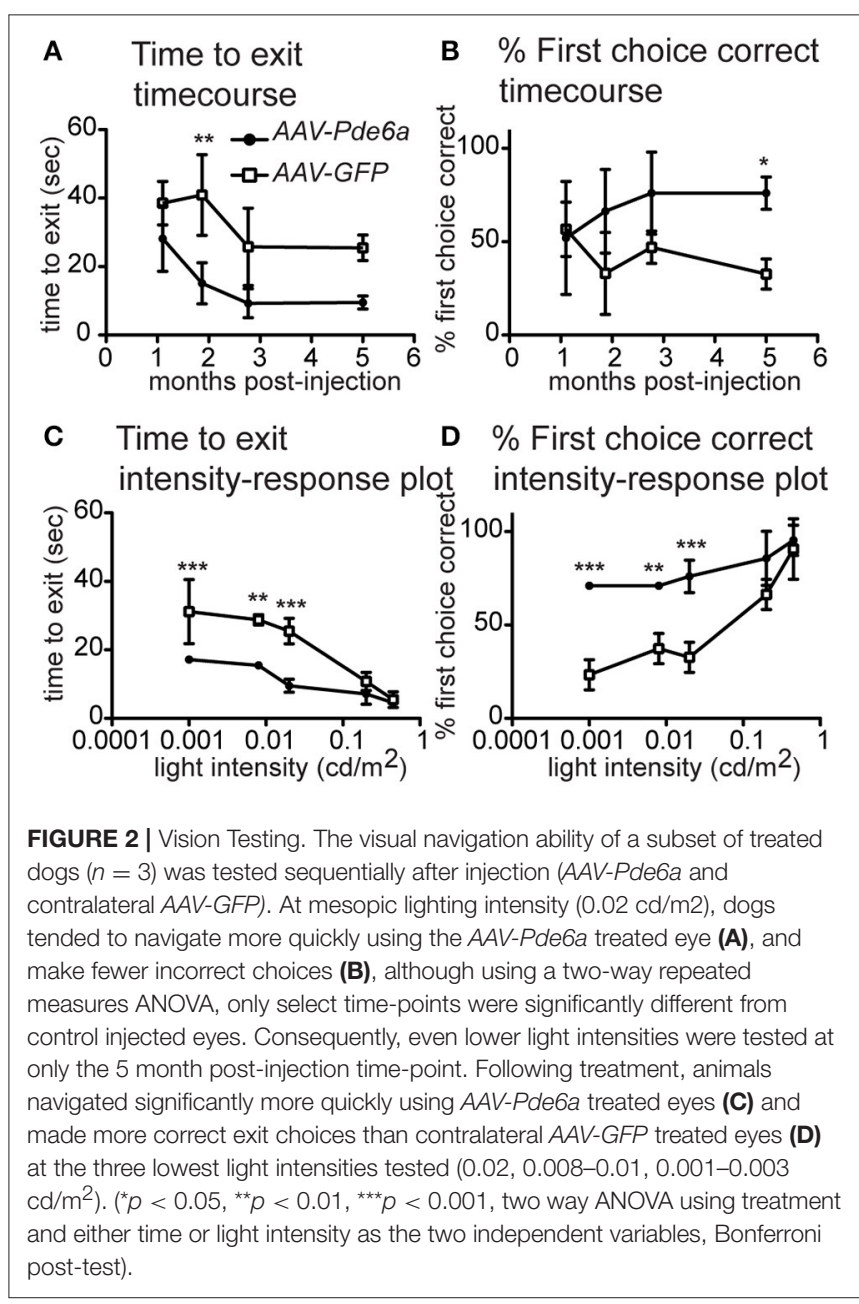




\section{Pde6a Gene Augmentation Results in Sustained Preservation of the Retinal Photoreceptor Nuclear Layer}

Resolution of the retinal bleb associated with subretinal injection occurred within $24 \mathrm{~h}$ in all injected eyes (Figures 3A,B). GFP expression was detected by fluorescent fundus imaging performed 1-month after injection in those eyes treated with $A A V-G F P$, and was sustained (data not shown). By 1 month following injection, mild arteriolar attenuation, which it typical of the Pde6a mutant dog natural history, was visible in untreated eyes, and ophthalmoscopic evidence suggestive of preservation of retinal thickness could be detected in the treated region in AAV-Pde6a treated eyes (Figures 3C,D). The degree of retinal thinning in the dog can be judged by the development of tapetal hyperreflectivity in the tapetal fundus and the injected regions subjectively were less hyperreflective than the adjacent untreated tapetal fundus. By 2 months following injection, significant arteriolar and mild venular attenuation was visible in untreated eyes (Figure 3E), and untreated regions of AAV-Pde6a treated eyes, compared with preservation of more normal vascular thickness in the treated area (Figure 3F).
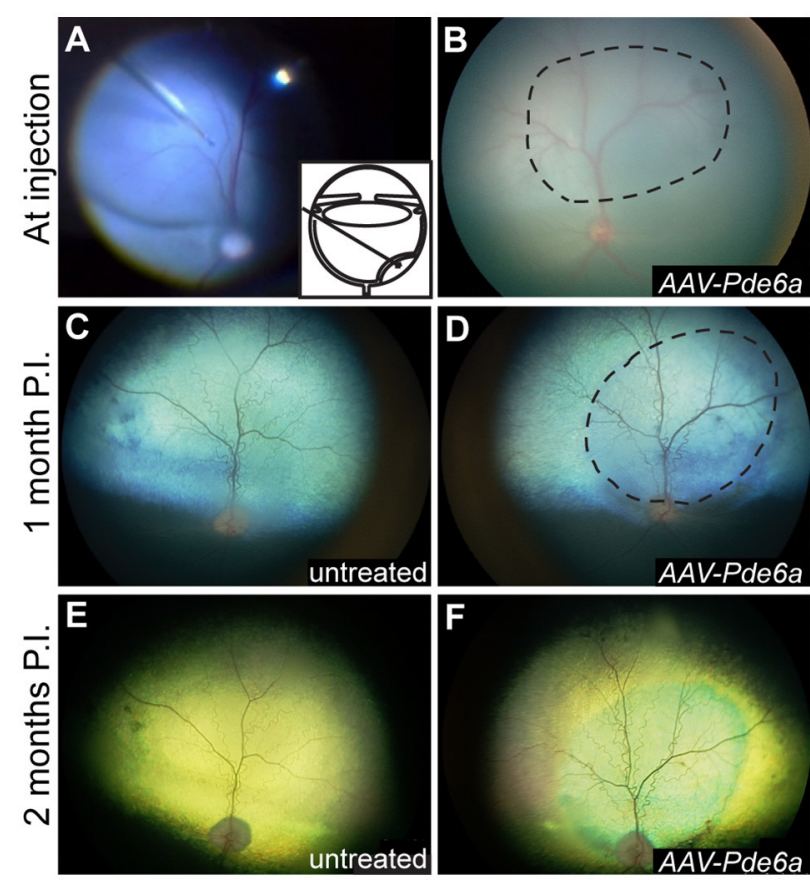

FIGURE 3 | Fundus photography. Subretinal injections of vectors were performed at 29-44 days of age; a representative intraoperative color photograph and schematic of the subretinal injection technique are shown in (A). The subretinal bleb often flattened significantly immediately after injection (B), and was visibly resolved within $24 \mathrm{~h}$ in all eyes. One month following injection, mild arteriolar attenuation was present in control, uninjected eyes (C), and mild preservation of normal arteriolar diameter was present in the treated region of $A A V-P d e 6 a$ injected eyes (D). By 2 months following injection untreated eyes and untreated regions of $A A V$-Pde6a treated eyes had significant arteriolar and mild venular attenuation (E), which contrasted with preserved arteriolar and venular vessel diameters in the treated area (F) Dashed line delineates the injected area.
SD-OCT was performed in three animals (12-046, 12-047, and 12-048). These animals were treated at 41-42 days of age; the contralateral eye was treated with $A A V-G F P$ (Table 1). $A A V$ $P d e 6 a$ treated eyes had relatively less thinning of the outer nuclear layer (ONL) throughout the 5 months following treatment compared with $A A V$-GFP treated eyes in which thinning of the ONL progressed more rapidly (Figure 4A). By 5 months following injection, the ONL in the AAV-Pde6a treated area was significantly thicker than that of the contralateral eye ( $A A V-G F P$

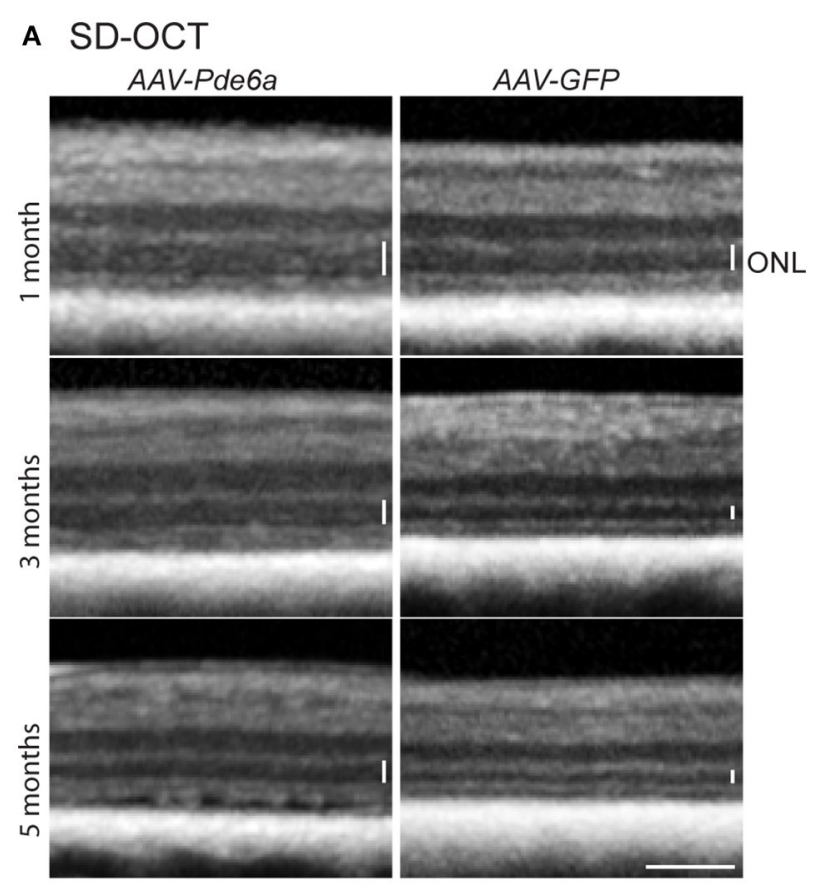

B Outer nuclear layer thickness

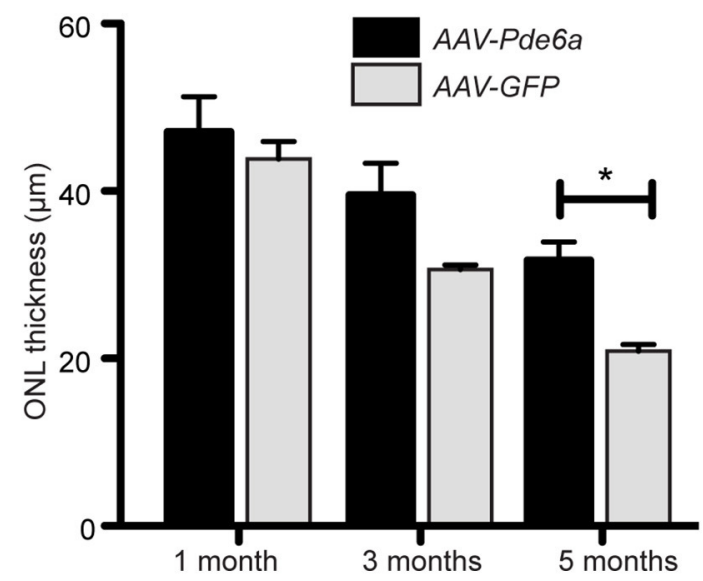

FIGURE 4 | Spectral domain optical coherence tomography. SD-OCT was performed on three animals injected with AAV-Pde6a at 41-42 days of age, contralateral eyes were injected with AAV-GFP. Animals were imaged at 1, 3, and 5 months post-treatment (A). The outer nuclear layer in AAV-Pde6a treated eyes was thicker compared with contralateral AAV-GFP treated eyes, and this difference was significant at 5 months post-injection (B). ${ }^{*} p<0.05$, two-way repeated measures ANOVA, scale bar represents $200 \mu \mathrm{m}$. 
treated) (Figure 4B). Note that in some of the gene therapy treated eyes retinal abnormalities developed as described further below. Careful assessment of the zones on SD-OCT showed that in AAV-Pde6a treated retinal regions there the region that represents the photoreceptor inner and outer segments have improved definition and indicated that the inner/outer segment length was greater than in the adjacent untreated region of the same eye and the in the comparable retinal region of the $A A V$-GFP treated eye (Supplementary Figure 1). Supplementary Figure 1 also shows a heat map of a $A A V-P d e 6 a$ treated retinal region showing photoreceptor preservation compared to the immediately adjacent untreated region of the same eye.

\section{AAV-Pde6a Treated Retinal Regions Demonstrate Outer Segment Pde6 Expression and More Appropriate Localization of Rhodopsin to the Outer Segment}

The single animal (12-061) examined at 2 weeks following injection, had obvious Pde6 protein readily detectable by IHC in the photoreceptor outer segments in the treated area of $A A V$ Pde6a injected eye (Figure 5A) compared with a low level of labeling in the $A A V$-GFP treated eye (Figure 5B). GFP expression was detected by IHC at 2 weeks following injection in $A A V$ GFP treated eyes (Figure 5B). In the animal (12-060) examined at 4 weeks a more marked difference in outer segment Pde6 expression was noted between the two eyes (Figures 5C,D), and only small amounts of remaining Pde6 were detected in some of the control injected eyes at this timepoint, Pde6 protein was not detected in control eyes at later timepoints. Pde6 protein expression was sustained for at least 3 months in the AAV-Pde6a injected region (Figure 5E). Rhodopsin IHC showed there was less mislocalization to the rod cell body in the AAV-Pde6a treated retinal regions and improved trafficking to the outer segments (Figures 5F,G). Pde6b expression was also detected in treated areas, but not in untreated areas (Figures 5H,I), suggesting that treatment helped stabilize the full Pde6 complex as the other subunits are secondarily deficient in this model (Tuntivanich et al., 2009). Very low levels of cyclic GMP (cGMP) were detectable by IHC in the injected area of the $A A V-P d e 6 a$ treated eye, but outside of the injected area, abnormally high levels of cGMP were present in the outer nuclear layer, corresponding with loss of outer nuclear cell layer nuclei and absence of Pde6 expression (Figures 5J-L). Cone photoreceptors in AAV-Pde6a treated areas appeared to have a normal morphology compared to those in the untreated areas which were stunted (Figures 5M,N). Glial fibrillary acidic protein (GFAP) expression within the retina was also lower in Pde6 expressing areas (Figure 5O). In untreated areas, GFAP positive filaments more frequently extended to the photoreceptor outer nuclear layer, indicating gliosis (Figure 5P). Rod bipolar cell dendritic arbors were maintained with a normal appearance in the treated retinal regions (Figure 5Q), whereas there was retraction of the dendritic arbors in the untreated retinal regions (Figure 5R).

\section{Pde6a Gene Augmentation Results in Prolonged Survival of Rod and Cone Photoreceptors}

The surviving nuclei of rods (Figure 5S) and cones (Figure 5T) were quantified in the two groups of treated animals (five animals treated at 30 days, contralateral eye untreated; three animals treated at 41-42 days, contralateral eye $A A V-G F P$ treated, Table 1). Rod photoreceptor nuclei in the AAV-Pde6a treated eye were significantly preserved in both groups, when compared to an analogous region in the contralateral eye (either untreated or AAV-GFP treated; Figure 5S). Cone photoreceptor numbers were not different between groups in animals treated at 30 days of age and maintained for 3 months, but cone nuclei were significantly preserved in animals treated with AAV-Pde6a at 4142 days and maintained for 5.5 months, compared with $A A V-G F P$ treated controls (Figure 5T). Comparison of rod numbers per unit retinal length were made between the two control groups (untreated and AAV-GFP injected) and there were no significant differences (data not shown).

\section{There Are Complications Associated with AAV-Mediated Gene Augmentation in Pde6a Mutant Dogs}

Five dogs were injected at 41-44 days of age. Two of these dogs were euthanized at 2 and 4 weeks respectively following injection, and not assessed for retinal thickness. In the remaining three dogs there was evidence of regions of retinal thinning within the treated areas on fundus images in both AAV-Pde6a (Figures 6A,B) and $A A V$-GFP treated eyes (Figures 6C,D). CSLO images show changes in AAV-Pde6a treated eyes (Figure 6C), and in fundus autofluorescence images of $A A V-G F P$ treated eyes, there were multifocal round to oval-shaped regions where fluorescence was diminished within the treated area, most likely due to the absence of cells expressing GFP in degenerate areas (Figure 6D). SD-OCT images showed that these regions represented either focal retinal detachment (Figure 6E, arrow), or marked thinning of the photoreceptor outer nuclear layer (Figure 6E, arrowhead). Histology of affected eyes demonstrated that the detached areas appeared to represent rosettes that contained outer segments of both cones (Figures 6F,G), and rods (Figures 6H,I). The rosettes were present predominantly within injected areas-surviving rod photoreceptors expressed Pde6 in $A A V$-Pde6a treated eyes (Figures 6F,G), and GFP in AAV-GFP treated eyes (data not shown).

\section{DISCUSSION}

$A A V$-Pde6a gene augmentation therapy in a small number of young Pde6a mutant dogs resulted in restoration of Pde6 protein in the outer segments of rods in all treated retinal areas. Restoration of Pde6 in the outer segments was associated with rod function on ERG in 5 of 9 eyes tested as evidenced by development of a scotopic threshold response and alterations in the timing of the b-wave. These same five treated eyes had improved dim-light vision compared to the control eyes. The scotopic threshold response is a rod mediated response (despite 

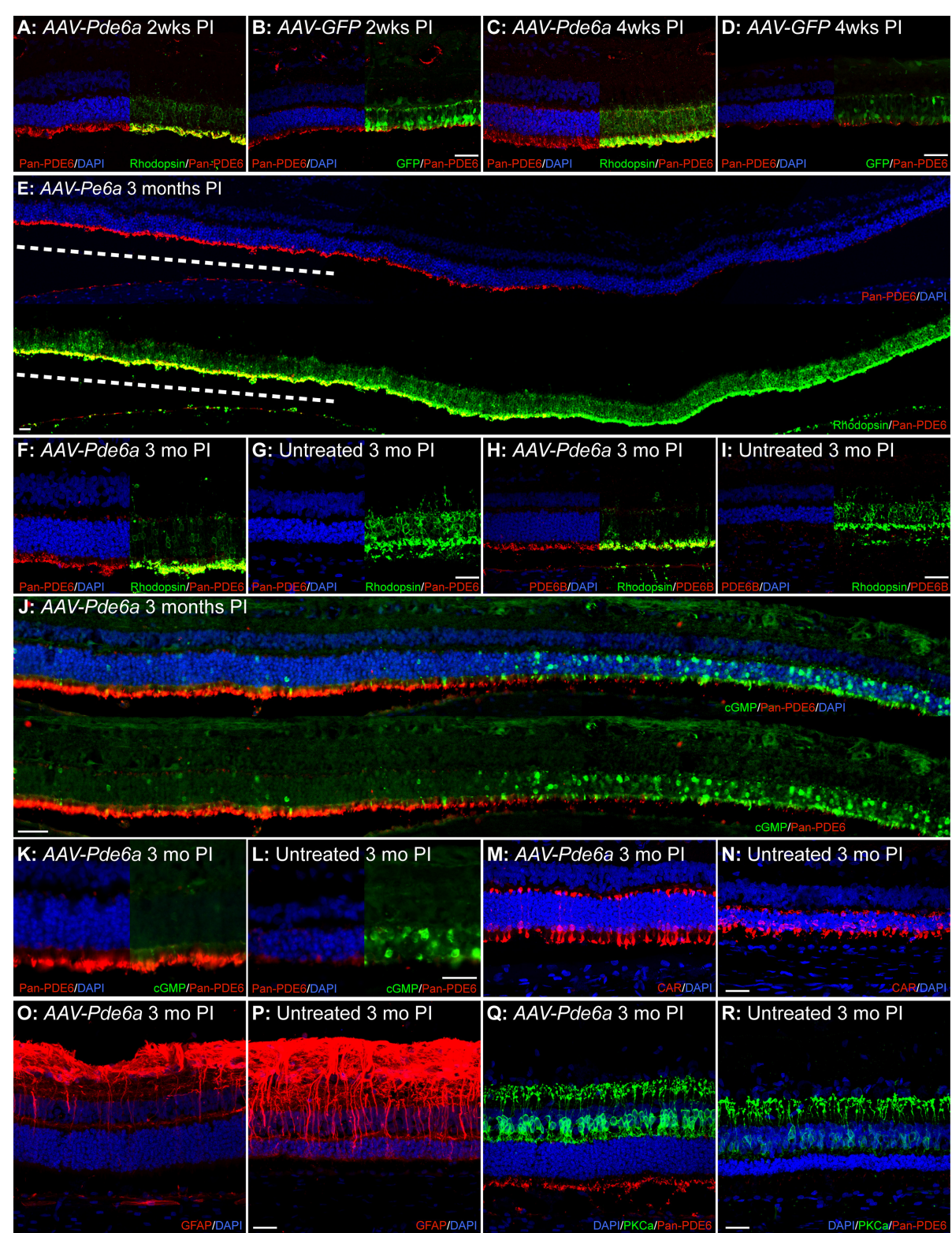

S: Rod photoreceptor numbers

T: Cone photoreceptor numbers

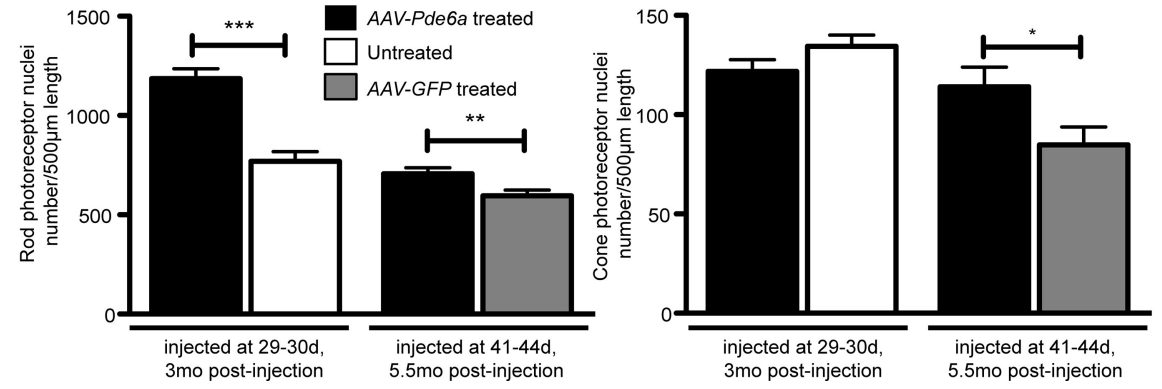

FIGURE 5 | Histological features of Pde6a gene augmentation treatment. Two weeks following injection increased immunolabeling for PDE6 protein was detected in the AAV-Pde6a treated eye (A), and GFP expression was detected at this stage in the contralateral AAV-GFP injected eye (B). PDE6 expression was sustained in the AAV-Pde6a treated eye after 4 weeks (C) but very little was detected in eyes treated with AAV-GFP (D). Three months following treatment with AAV-Pde6a, outer segment detection of PDE6 was sustained in the injected area (dashed white line; $\mathbf{E}$ ), and there was reduction in the amount of rhodopsin mislocalized into the rod cell bodies in the treated region (F) compared with significant cell body mislocalization in untreated contralateral eyes (G). Pde6b, a subunit not directly delivered by 


\section{FIGURE 5 | Continued}

treatment with AAV-Pde6a, was also detected in the rod outer segments of AAV-Pde6a treated eyes $\mathbf{( H )}$ compared with minimal signal in untreated contralateral eyes (I). Cyclic GMP levels were reduced in the injected area of eyes treated with AAV-Pde6a (J,K), but was detected at much higher levels in outer nuclear cell bodies in untreated regions of the same eyes and also in untreated eyes (L). Cone inner and outer segment morphology was better preserved in $A A V-P d e 6 a$ treated eyes (M) compared with untreated eyes (N). GFAP positive fibrils extending into the outer nuclear layer were only detected in a few glia in $A A V$-Pde6a treated eyes (O) compared with significant numbers in untreated eyes $\mathbf{( P )}$. The rod bipolar dendrites appeared to have a normal distribution in the treated retinal region ( $\mathbf{Q})$ whereas by this age there was clear dendrite retraction in the untreated retinal regions (R). In both earlier and later treated groups rod photoreceptor nuclei numbers in treated areas of $A A V$-Pde6a injected eyes were significantly higher than matched regions in the contralateral eye (S). Cone numbers were not preserved in eyes treated early and harvested 3 months following injection compared with untreated controls, but there were significantly more cones surviving 5.5 months after later treatment with AAV-Pde6a, compared with AAV-GFP treated controls (T). CAR, cone arrestin; GFAP, glial fibrillary acidic protein; PKCa, protein kinase C-alpha; DAPI,

$4^{\prime}$,6-diamidino-2-phenylindole; PI, post-injection. Scale bars in all images represent $25 \mu \mathrm{m} .{ }^{\star} p<0.05$, ${ }^{\star \star} p<0.01$, ${ }^{\star \star \star} p<0.001$, paired $t$-test. Confocal microscopy images for all except (J-L) (standard fluorescence microscopy).
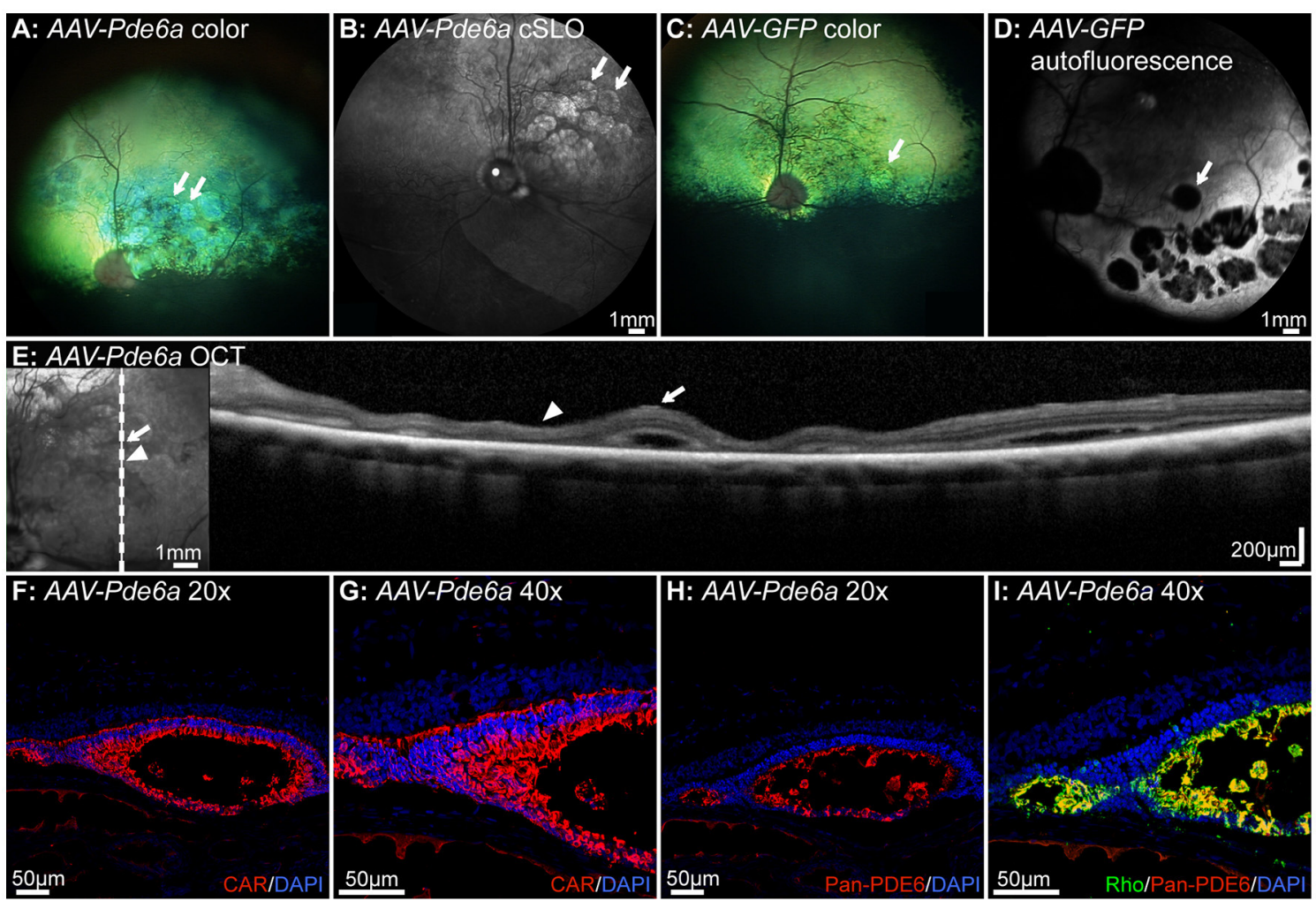

FIGURE 6 | Complications associated with gene therapy administration in Pde6a mutant dogs. In the 3 dogs injected at 41-42 days of age and injected bilaterally (one eye with $A A V-P d e 6 a$, one eye with $A A V-G F P$ ), changes on fundus images indicating retinal thinning were observed both in eyes injected with $A A V-P d e 6 a$ (A,B) and with AAV-GFP (C,D). Eyes treated with AAV-GFP had a hyperfluorescent area delineating the injected region, and contained within that areas of focal hypofluorescence, possibly indicating loss of GFP fluorescence or cell loss (D). White arrows delineate the corresponding areas of abnormality in (A-D). SD-OCT imaging of these areas (E) showed foci of retinal separation (white arrow) interspersed with areas of retinal thinning, predominantly of the outer nuclear layer (white arrowhead). Histologically, the areas of retinal separation appeared to be primitive rosettes, with cone cell bodies lining a lumen (F,G) containing cone and rod outer segments expressing transgenic Pde6 (H) and endogenous rhodopsin (I). CAR: cone arrestin, DAPI, 4',6-diamidino-2-phenylindole; Rho, rhodopsin.

the measured electrical signal originating in the inner retina) and appears at a stimulus luminance close to visual threshold (Sieving et al., 1986; Naarendorp and Sieving, 1991). The slow negative STR waveform initially increases in amplitude with increasing stimulus luminance but becomes obscured by the incursion of the b-wave. Previous studies in models have suggested that the STR threshold tracks remaining functional vision better than the ERG b-wave (Bush et al., 1995) The scotopic b-wave in the eyes that showed rescue had a longer implicit time, more in keeping with a rod-response, although the flash luminance needed to induce an ERG b-wave was not reduced by treatment. Previous ERG studies in dogs have shown that the rod-mediated b-wave is a slower waveform than the contribution of darkadapted cones to the scotopic responses to stronger stimuli that in normal dogs result in a mixed rod/cone response (Narfström et al., 1995) Pde6a mutant dogs that have not been treated, have no evidence of rod ERG responses and exhibit cone-only function (Tuntivanich et al., 2009). The small delayed (compared 
to control eyes), dark-adapted b-wave in treated eyes reflects a low level of functional rod rescue even allowing for the fact that only a proportion of the retina was treated (blebs typically extended over one-fifth to one-third of the retina). The darkadapted b-wave amplitudes recorded might also be low because (1) only a proportion of rods in the treated area are functional, or (2) the transduced rods have short outer segments reducing the individual response from each rod, or (3) rod bipolar cell dendritic connections to rods may have been reduced (as reported in other PDE6 models; Strettoi and Pignatelli, 2000), or a combination of these possibilities. The increase in dark-adapted b-wave implicit time combined with the presence of an STR, improved dim light vision, Pde6 localization to outer segments on IHC and photoreceptor structural preservation, provides convincing evidence of $A A V$-Pde6a mediated rod rescue.

Improvement in visual performance is reported to be a more sensitive measure of visual function than ERG. For example, in RPE65 gene augmentation therapy for Leber Congenital Amaurosis improved visual function can be detected in the absence of an ERG improvement both in human patients in phase I/II clinical trials, but also in the dog model when receiving a low dose of therapeutic vector (Bainbridge et al., 2008, 2015; Hauswirth et al., 2008; Maguire et al., 2008). This difference between the sensitivity of visual function testing and ERG likely results from a difference in the number of functional photoreceptors that are required for improvement in vision vs. the number required to create a large enough electrical signal to be detectable by a corneal electrode. Transgene mediated expression of Pde6 was sufficient to slow the typically very rapid loss of photoreceptors in the Pde6a mutant dog. Pde6 that formed in outer segments in the treated region was able to hydrolyze cGMP as evidenced by the large reduction of cGMP detectable by IHC restricted to the retinal regions that had Pde6 expression. Improved trafficking of rhodopsin to the outer segments in treated retinal regions largely reversed the mislocalization that occurred in untreated and control treated retinal regions. Rhodopsin mislocalization is a common finding in diseased and stressed rod photoreceptors (see Hollingsworth and Gross, 2012 for a review). Structural preservation of the outer nuclear layer was detectable both on SD-OCT, where it corresponded with the topography of the treated region, and in retinal sections where it corresponded with the region of increased Pde6 expression. There also appeared to be a positive effect on rod to bipolar cell synapses. In untreated retinal regions rod bipolar cell dendritic retraction was apparent. Treatment appeared to reverse, or prevent this change.

These results show that some rescue of rod function and rod preservation is possible in this severe retinal degeneration model. Previously we had not been able to achieve rescue using AAV5 vectors and hypothesized that the faster transduction rate achieved by AAV8 vector with a capsid mutation might allow us to achieve rescue, i.e., AAV8(Y733F). The greater success with an AAV8(Y733F) construct over an AAV5 serotype delivering the same construct (Petersen-Jones, unpublished findings) is in contrast to the findings in the Pde6b dog, which also has a rapid photoreceptor degeneration, where results with an AAV5 vector were similar to that achieved with AAV8 (Petit et al.,
2012). Conversely in rodent studies the stronger expression achieved by an AAV8 vector and an AAV8(Y733F) over AAV5 has been shown to allow for rescue of some of the more severe photoreceptor degeneration phenotypes such as the Aipl1-/mouse model, that has a very rapid retinal degeneration (Sun et al., 2010) and also the Pde6b $b^{r d 10}$ mouse model which has a slower photoreceptor degeneration (Pang et al., 2011). We had also previously shown in a reporter gene study that an AAV8(Y733F) vector had a stronger and faster onset of reporter gene expression compared to AAV5 vectors when delivered by subretinal injection in the dog (Mowat et al., 2014).

Pde6a mutant dogs, in addition to lacking detectable Pde6a on Western blot analysis of pre-degenerate retinal tissue, also lack the other Pde6 subunits (Pde6b and Pde6g) (Tuntivanich et al., 2009). This is in contrast to the findings in Pde6b mutant dogs where the other Pde6 subunits were detectable on Western blotting of retinal tissue (Suber et al., 1993). It is not clear whether the lack of the other Pde6 subunits in Pde6a mutant dogs explains the greater difficulty we have faced in achieving rescue compared with the results reported for the Pde6b mutant dog (Petit et al., 2012; Pichard et al., 2016).

Based on these promising preliminary gene augmentation therapy results using AAV8(Y733F) to deliver Pde6a under control of a ubiquitous promoter further studies are warranted to optimize the degree of rescue and minimize adverse effects. This could include optimization of construct with respect to promoter to specifically target rods and possibly other modifications such as addition of an enhancer element or mini-intron. The Pde6a cDNA is too large to allow packaging in a self-complementary vector which could otherwise hasten the onset of expression (Petersen-Jones et al., 2009). A dose escalation study is also needed to establish the optimal therapeutic dose. It is conceivable that the variability of the degree of rescue achieved could mean that the current dose is on the edge of the most effective range, either giving too little expression, meaning that the threshold for rescue was not achieved in each eye, or too high a level of expression resulting in a range of adverse effects. With a condition with such a rapid degeneration the timing of the injection is also likely to be important and that to improve results younger animals with a larger number of surviving rods should be targeted. However, in our study more consistent results were achieved by injecting slightly older animals. Visualization in younger animals during the injection can be challenging because the ocular media is not always completely clear and remnants of development vasculature (pupillary membrane, tunica vasculosa lentis, and hyaloid vasculature) are often present. Thus, obtaining a good subretinal bleb in younger animals presents a technical challenge thus limiting the lower end of the age range that can be treated efficiently. In mouse Pde6 models, introduction of expression of the missing Pde6 subunit achieved rescue even with more advanced loss of photoreceptors (Wert et al., 2013; Koch et al., 2015). Although unlike the Pde6a mutant dog neither model had a complete absence of Pde6 in the pre-degenerate retina.

Limitations of this study include the necessarily small sample size due to utilization of a large animal model species. Statistical evaluation was therefore limited in many 
comparisons, particularly as half of the animals underwent no treatment in the contralateral control eye, the other half of the animals were injected with a control vector, more suitable to compare to the treated $A A V$-Pde6a treated eyes, as the effects of injection are controlled. We chose both untreated and control vector treated controls to allow for possible adverse effects of subretinal injection and expression of the GFP reporter gene and conversely possible trophic factor release due to the same interference. The titer of AAVGFP used was one that would not be expected to lead to loss of photoreceptors due to high expression levels of GFP. Due to limitations of equipment availability in the laboratory at the time of the experiments, certain studies could not be performed on both cohorts of animals limiting the full evaluation of outcome of therapy. Clearly, further work is necessary to expand on these promising initial results aiming to optimize treatment efficacy, and future experiments would consider these factors.

The gene augmentation therapy in this study was not without complications. Adverse retinal changes occurred with both the $A A V$-Pde $6 a$ and the $A A V$-GFP vectors. In some eyes this resulted in focal lesions of severe retinal thinning within the injected region. These degenerate lesions occurred directly adjacent to other retinal areas where transgene expression was present and where in $A A V$-Pde6a injected eyes but not the $A A V$-GFP injected eyes there was retinal preservation. Retinal folds and rosettes were also apparent in some eyes. Retinal lesions and inflammation following subretinal AAV vectors in dogs has previously been described. Bainbridge et al., reported that 3 out of 8 dogs that were treated by subretinal injection of an AAV2 construct expressing GFP under control of a CMV promoter developed delayed ocular inflammation (Bainbridge et al., 2003). Beltran et al found that some dogs developed patches of retinal degeneration and more severe inflammatory response following subretinal administration of an AAV5 expressing GFP under different promoters (Beltran et al., 2010). In our study, no signs of retinal inflammation were detected in any eye at any timepoint, either in vivo or histologically. In the current study dogs were treated prior to retinal maturation, which in the dog is considered to be complete at about 7-8 weeks of age (Gum et al., 1984). It is not clear if this might be a factor in rosette formation or whether that change might be associated with the retinal detachment that is induced by the subretinal injection. Similar rosettes and areas of retinal thinning were reported in a safety study of an $A A V-R P E 65$ vector in adult dogs. The effect was dose related, with development of lesions occurring in eyes injected with a higher vector dose (Jacobson et al., 2006). A similar gene therapy study in which Pde6b mutant dogs were injected at 20 days of age with AAV vectors expressing Pde6b under control of a tissue specific promoter (Rhodopsin Kinase) to target photoreceptors did not report similar rosette formation (Petit et al., 2012). In contrast, our study used a ubiquitous promoter so off target effects are a possibility, although we did not identify Pde6 protein presence outside of the rod outer segments. It is important to note that within the treated retinal regions the retina adjacent to the degenerate lesions showed Pde6 localization to the outer segments and preservation of the outer nuclear layer. The process of performing a subretinal injection causes a temporary retinal detachment which can result in deleterious effects (Cook et al., 1995; Fisher et al., 2001; Fisher and Lewis, 2003). These could potentially be even more damaging in a diseased retina. Complications potentially associated with subretinal injections have also been reported in RPE65 clinical trials, including foveal thinning and development of a macular hole (Maguire et al., 2008; Jacobson et al., 2012).

Here we report that AAV-mediated gene augmentation in a large animal model of rapid onset arRP caused by a Pde6a mutation results in Pde6 expression in rod photoreceptor outer segments restores a degree of rod photoreceptor function, delays photoreceptor degeneration, and reduces rhodopsin mislocalization, GFAP activation and bipolar cell dendrite retraction. Future studies will be designed to improve the degree and duration of rescue and to avoid adverse treatment effects that were associated with the therapy. These additional studies will be important prior to translation of this early proof-of-concept functional success with the Pde6a mutant dog model into clinical trials for human arRP.

\section{AUTHOR CONTRIBUTIONS}

FM: Evaluation of retinal samples and immunohistochemistry, data interpretation and wrote the paper. LO: SD-OCT analysis and some IHC, review of paper. JB: Helped in design, assisted in subretinal injections, critical review of manuscript. KG: Performed outcome measures and data analysis, reviewed manuscript. AB: Measurement of retinal sections and cells, reviewed manuscript. JQ: Assisted in all animal experiments and performed ERG and vision testing. $\mathrm{AD}$ : Design and production of viral vectors. SB: Design of vector approach and production of viral vectors, critical review of manuscript. WH: Design of vectors, production of viral vectors, critical review of manuscript. SP: Designed and oversaw experiments, subretinal injections, co-wrote the manuscript.

\section{FUNDING}

Funding from NIH grant R01EY014160 and Myers Dunlap Endowment to SP. Midwest Eye Banks to JB. NIH P30EY021721 to WH, Macula Vision Research Foundation to WH, an unrestricted grant from Research to Prevent Blindness Inc. to the University of Florida Department of Ophthalmology.

\section{SUPPLEMENTARY MATERIAL}

The Supplementary Material for this article can be found online at: http://journal.frontiersin.org/article/10.3389/fnins. 2017.00342/full\#supplementary-material 


\section{REFERENCES}

Aguirre, G. D., Farber, D., Lolley, R., Fletcher, R. T., and Chader, G. J. (1978). Rodcone dysplasia in Irish setters: a defect in cyclic GMP metabolism in visual cells. Science 201, 1133-1134. doi: 10.1126/science.210508

Annear, M. J., Bartoe, J. T., Barker, S. E., Smith, A. J., Curran, P. G., Bainbridge, J. W., et al. (2011). Gene therapy in the second eye of RPE65-deficient dogs improves retinal function. Gene Ther. 18, 53-61. doi: 10.1038/gt.2010.111

Bainbridge, J. W., Mehat, M. S., Sundaram, V., Robbie, S. J., Barker, S. E., Ripamonti, C., et al. (2015). Long-term effect of gene therapy on Leber's congenital amaurosis. N. Engl. J. Med. 372, 1887-1897. doi: 10.1056/NEJMoa1414221

Bainbridge, J. W., Mistry, A., Schlichtenbrede, F. C., Smith, A., Broderick, C., De Alwis, M., et al. (2003). Stable rAAV-mediated transduction of rod and cone photoreceptors in the canine retina. Gene Ther. 10, 1336-1344. doi: $10.1038 /$ sj.gt.3301990

Bainbridge, J. W., Smith, A. J., Barker, S. S., Robbie, S., Henderson, R., Balaggan, K., et al. (2008). Effect of gene therapy on visual function in Leber's congenital amaurosis. N. Engl. J. Med. 358, 2231-2239. doi: 10.1056/NEJMoa0802268

Barhoum, R., Martinez-Navarrete, G., Corrochano, S., Germain, F., FernandezSanchez, L., de la Rosa, E. J., et al. (2008). Functional and structural modifications during retinal degeneration in the rd10 mouse. Neuroscience 155 , 698-713. doi: 10.1016/j.neuroscience.2008.06.042

Beltran, W. A., Boye, S. L., Boye, S. E., Chiodo, V. A., Lewin, A. S., Hauswirth, W. W., et al. (2010). rAAV2/5 gene-targeting to rods:dose-dependent efficiency and complications associated with different promoters. Gene Ther. 17, 1162-1174. doi: $10.1038 /$ gt.2010.56

Bennett, J., Tanabe, T., Sun, D., Zeng, Y., Kjeldbye, H., Gouras, P., et al. (1996). Photoreceptor cell rescue in retinal degeneration (rd) mice by in vivo gene therapy. Nat. Med. 2, 649-654. doi: 10.1038/nm0696-649

Bush, R. A., Hawks, K. W., and Sieving, P. A. (1995). Preservation of inner retinal responses in the aged Royal College of Surgeons rat. Evidence against glutamate excitotoxicity in photoreceptor degeneration. Invest. Ophthalmol. Vis. Sci. 36, 2054-2062.

Chang, B., Hawes, N. L., Pardue, M. T., German, A. M., Hurd, R. E., Davisson, M. T., et al. (2007). Two mouse retinal degenerations caused by missense mutations in the beta-subunit of rod cGMP phosphodiesterase gene. Vision Res. 47, 624-633. doi: 10.1016/j.visres.2006.11.020

Cideciyan, A. V., Aleman, T. S., Boye, S. L., Schwartz, S. B., Kaushal, S., Roman, A. J., et al. (2008). Human gene therapy for RPE65 isomerase deficiency activates the retinoid cycle of vision but with slow rod kinetics. Proc. Natl. Acad. Sci. U.S.A. 105, 15112-15117. doi: 10.1073/pnas.0807027105

Cideciyan, A. V., Hauswirth, W. W., Aleman, T. S., Kaushal, S., Schwartz, S. B., Boye, S. L., et al. (2009a). Human RPE65 Gene therapy for leber congenital amaurosis: persistence of early visual improvements and safety at 1 Year. Hum. Gene Ther. 20, 999-1004. doi: 10.1089/hum.2009.086

Cideciyan, A. V., Hauswirth, W. W., Aleman, T. S., Kaushal, S., Schwartz, S. B., Boye, S. L., et al. (2009b). Vision 1 year after gene therapy for Leber's congenital amaurosis. N. Engl. J. Med. 361, 725-727. doi: 10.1056/NEJMc0903652

Cook, B., Lewis, G. P., Fisher, S. K., and Adler, R. (1995). Apoptotic photoreceptor degeneration in experimental retinal detachment. Invest. Ophthalmol. Vis. Sci. 36, 990-996.

Davis, R. J., Tosi, J., Janisch, K. M., Kasanuki, J. M., Wang, N. K., Kong, J., et al. (2008). Functional rescue of degenerating photoreceptors in mice homozygous for a hypomorphic cGMP phosphodiesterase $6 \mathrm{~b}$ allele (Pde6bH620Q). Invest. Ophthalmol. Vis. Sci. 49, 5067-5076. doi: 10.1167/iovs.07-1422

Dryja, T. P., Rucinski, D. E., Chen, S. H., and Berson, E. L. (1999). Frequency of mutations in the gene encoding the alpha subunit of rod cGMP-phosphodiesterase in autosomal recessive retinitis pigmentosa. Invest. Ophthalmol. Vis. Sci. 40, 1859-1865.

Farber, D. B. (1995). From mice to men: the cyclic GMP phosphodiesterase gene in vision and disease. The proctor lecture. Invest. Ophthalmol. Vis. Sci. 36, 263-275.

Farber, D. B., Danciger, J. S., and Aguirre, G. (1992). The beta subunit of cyclic GMP phosphodiesterase mRNA is deficient in canine rod-cone dysplasia 1. Neuron 9, 349-356. doi: 10.1016/0896-6273(92)90173-B

Fisher, S. K., and Lewis, G. P. (2003). Muller cell and neuronal remodeling in retinal detachment and reattachment and their potential consequences for visual recovery: a review and reconsideration of recent data. Vision Res. 43, 887-897. doi: 10.1016/S0042-6989(02)00680-6

Fisher, S. K., Stone, J., Rex, T. S., Linberg, K. A., and Lewis, G. P. (2001). Experimental retinal detachment: a paradigm for understanding the effects of induced photoreceptor degeneration. Prog. Brain Res. 131, 679-698. doi: 10.1016/S0079-6123(01)31053-1

Gargini, C., Terzibasi, E., Mazzoni, F., and Strettoi, E. (2007). Retinal organization in the retinal degeneration 10 (rd10) mutant mouse: a morphological and ERG study. J. Comp. Neurol. 500, 222-238. doi: 10.1002/cne.21144

Gearhart, P. M., Gearhart, C. C., and Petersen-Jones, S. M. (2008). A novel method for objective vision testing in canine models of inherited retinal disease. Invest. Ophthalmol. Vis. Sci. 49, 3568-3576. doi: 10.1167/iovs.07-0625

Gum, G. G., Gelatt, K. C., and Samuelson, D. A. (1984). Maturation of the retina of the canine neonate as determined by electroretinography and histology. Am. J. Vet. Res. 45, 1166-1171.

Guo, X., Wang, S. B., Xu, H., Ribic, A., Mohns, E. J., Zhou, Y., et al. (2015). A short N-terminal domain of HDAC4 preserves photoreceptors and restores visual function in retinitis pigmentosa. Nat. Commun. 6:8005. doi: $10.1038 /$ ncomms 9005

Hackam, A. S., Strom, R., Liu, D., Qian, J., Wang, C., Otteson, D., et al. (2004). Identification of gene expression changes associated with the progression of retinal degeneration in the rd1 mouse. Invest. Ophthalmol. Vis. Sci. 45, 2929-2942. doi: 10.1167/iovs.03-1184

Hart, A. W., McKie, L., Morgan, J. E., Gautier, P., West, K., Jackson, I. J., et al. (2005). Genotype-phenotype correlation of mouse pde6b mutations. Invest. Ophthalmol. Vis. Sci. 46, 3443-3450. doi: 10.1167/iovs.05-0254

Hartong, D. T., Berson, E. L., and Dryja, T. P. (2006). Retinitis pigmentosa. Lancet 368, 1795-1809. doi: 10.1016/S0140-6736(06)69740-7

Hauswirth, W. W., Aleman, T. S., Kaushal, S., Cideciyan, A. V., Schwartz, S. B., Wang, L. L., et al. (2008). Treatment of leber congenital amaurosis due to RPE65 mutations by ocular subretinal injection of adeno-associated virus gene vector: short-term results of a phase I trial. Hum. Gene Ther. 19, 979-990. doi: 10.1089/hum.2008.107

Hauswirth, W. W., Lewin, A. S., Zolotukhin, S., and Muzyczka, N. (2000). Production and purification of recombinant adeno-associated virus. Methods Enzymol. 316, 743-761. doi: 10.1016/S0076-6879(00)16760-6

Hollingsworth, T. J., and Gross, A. K. (2012). Defective trafficking of rhodopsin and its role in retinal degenerations. Int. Rev. Cell Mol. Biol. 293, 1-44. doi: 10.1016/B978-0-12-394304-0.00006-3

Jacobson, S. G., Acland, G. M., Aguirre, G. D., Aleman, T. S., Schwartz, S. B., Cideciyan, A. V., et al. (2006). Safety of recombinant adeno-associated virus type 2-RPE65 vector delivered by ocular subretinal injection. Mol. Ther. 13, 1074-1084. doi: 10.1016/j.ymthe.2006.03.005

Jacobson, S. G., Cideciyan, A. V., Ratnakaram, R., Heon, E., Schwartz, S. B., Roman, A. J., et al. (2012). Gene therapy for leber congenital amaurosis caused by RPE65 mutations: safety and efficacy in 15 children and adults followed up to 3 years. Arch. Ophthalmol. 130, 9-24. doi: 10.1001/archophthalmol.2011.298

Jomary, C., Vincent, K. A., Grist, J., Neal, M. J., and Jones, S. E. (1997). Rescue of photoreceptor function by AAV-mediated gene transfer in a mouse model of inherited retinal degeneration. Gene Ther. 4, 683-690. doi: $10.1038 /$ sj.gt. 3300440

Keeler, C. (1966). Retinal degeneration in the mouse is rodless retina. J. Hered. 57, 47-50. doi: 10.1093/oxfordjournals.jhered.a107462

Koch, S. F., Tsai, Y. T., Duong, J. K., Wu, W. H., Hsu, C. W., Wu, W. P., et al. (2015). Halting progressive neurodegeneration in advanced retinitis pigmentosa. $J$. Clin. Invest. 125, 3704-3713. doi: 10.1172/JCI82462

Ku, C. A., Chiodo, V. A., Boye, S. L., Hayes, A., Goldberg, A. F., Hauswirth, W. W., et al. (2015). Viral-mediated vision rescue of a novel AIPL1 cone-rod dystrophy model. Hum. Mol. Genet. 24, 670-684. doi: 10.1093/hmg/ddu487

Kumar-Singh, R., and Farber, D. B. (1998). Encapsidated adenovirus minichromosome-mediated delivery of genes to the retina: application to the rescue of photoreceptor degeneration. Hum. Mol. Genet. 7, 1893-1900. doi: $10.1093 / \mathrm{hmg} / 7.12 .1893$

Li, A., Zhu, X., Brown, B., and Craft, C. M. (2003). Gene expression networks underlying retinoic acid-induced differentiation of human retinoblastoma cells. Invest. Ophthalmol. Vis. Sci. 44, 996-1007. doi: 10.1167/iovs.02-0434

Maguire, A. M., High, K. A., Auricchio, A., Wright, J. F., Pierce, E. A., Testa, F., et al. (2009). Age-dependent effects of RPE65 gene therapy for Leber's 
congenital amaurosis: a phase 1 dose-escalation trial. Lancet 374, 1597-1605. doi: 10.1016/S0140-6736(09)61836-5

Maguire, A. M., Simonelli, F., Pierce, E. A., Pugh, E. N. Jr., Mingozzi, F., Bennicelli, J., et al. (2008). Safety and efficacy of gene transfer for Leber's congenital amaurosis. N. Engl. J. Med. 358, 2240-2248. doi: 10.1056/NEJMoa0802315

McLaughlin, M. E., Ehrhart, T. L., Berson, E. L., and Dryja, T. P. (1995). Mutation spectrum of the gene encoding the beta subunit of rod phosphodiesterase among patients with autosomal recessive retinitis pigmentosa. Proc. Natl. Acad. Sci. U.S.A. 92, 3249-3253. doi: 10.1073/pnas.92.8.3249

Mowat, F. M., Breuwer, A. R., Bartoe, J. T., Annear, M. J., Zhang, Z., Smith, A. J., et al. (2013). RPE65 gene therapy slows cone loss in Rpe65-deficient dogs. Gene Ther. 20, 545-555. doi: 10.1038/gt.2012.63

Mowat, F. M., Gornik, K. R., Dinculescu, A., Boye, S. L., Hauswirth, W. W., Petersen-Jones, S. M., et al. (2014). Tyrosine capsid-mutant AAV vectors for gene delivery to the canine retina from a subretinal or intravitreal approach. Gene Ther. 21, 96-105. doi: 10.1038/gt.2013.64

Naarendorp, F., and Sieving, P. A. (1991). The scotopic threshold response of the cat ERG is suppressed selectively by GABA and glycine. Vis. Res. 31, 1-15. doi: 10.1016/0042-6989(91)90068-G

Narfström, K., Andersson, B. E., Andreasson, S., and Gouras, P. (1995). Clinical electroretinography in the dog with ganzfeld stimulation: a practical method of examining rod and cone function. Doc. Ophthalmol. 90, 279-290. doi: 10.1007/BF01203863

Nishiguchi, K. M., Carvalho, L. S., Rizzi, M., Powell, K., Holthaus, S. M., Azam, S. A., et al. (2015). Gene therapy restores vision in rd1 mice after removal of a confounding mutation in Gpr179. Nat. Commun. 6, 6006. doi: $10.1038 /$ ncomms7006

Pang, J. J., Boye, S. L., Kumar, A., Dinculescu, A., Deng, W., Li, J., et al. (2008). AAV-mediated gene therapy for retinal degeneration in the rd10 mouse containing a recessive PDE $\beta$ mutation. Invest. Ophthalmol. Vis. Sci. 49, 4278-4283. doi: 10.1167/iovs.07-1622

Pang, J. J., Dai, X., Boye, S. E., Barone, I., Boye, S. L., Mao, S., et al. (2011). Longterm retinal function and structure rescue using capsid mutant AAV8 vector in the rd10 mouse, a model of recessive retinitis pigmentosa. Mol. Ther. 19, 234-242. doi: 10.1038/mt.2010.273

Petersen-Jones, S. M., Bartoe, J. T., Fischer, A. J., Scott, M., Boye, S. L., Chiodo, V., et al. (2009). AAV retinal transduction in a large animal model species: comparison of a self-complementary AAV2/5 with a single-stranded AAV2/5 vector. Mol. Vis. 15, 1835-1842.

Petersen-Jones, S. M., Entz, D. D., and Sargan, D. R. (1999). cGMP phosphodiesterase- $\alpha$ mutation causes progressive retinal atrophy in the Cardigan Welsh corgi dog. Invest. Ophthalmol. Vis. Sci. 40, 1637-1644.

Petit, L., Lheriteau, E., Weber, M., Le Meur, G., Deschamps, J. Y., Provost, N., et al. (2012). Restoration of vision in the pde6 $\beta$-deficient dog, a large animal model of rod-cone dystrophy. Mol. Ther. 20, 2019-2030. doi: 10.1038/mt.2012.134

Petrs-Silva, H., Dinculescu, A., Li, Q., Deng, W. T., Pang, J. J., Min, S. H., et al. (2011). Novel properties of tyrosine-mutant AAV2 vectors in the mouse retina. Mol. Ther. 19, 293-301. doi: 10.1038/mt.2010.234

Pichard, V., Provost, N., Mendes-Madeira, A., Libeau, L., Hulin, P., Tshilenge, K. T., et al. (2016). AAV-mediated Gene therapy halts retinal degeneration in PDE6 $\beta$-deficient Dogs. Mol. Ther. 24, 867-876. doi: 10.1038/mt. 2016.37

Pittler, S. J., and Baehr, W. (1991). Identification of a nonsense mutation in the rod photoreceptor cGMP phophodiesterase á-subunit gene of the rd mouse. Proc. Natl. Acad. Sci. U.S.A. 88, 8322-8326. doi: 10.1073/pnas.88.19.8322

Pittler, S. J., Keeler, C. E., Sidman, R. L., and Baehr, W. (1993). PCR analysis of DNA from 70-year-old sections of rodless retina demonstrates identity with the mouse rd defect. Proc. Natl. Acad. Sci. U.S.A. 90, 9616-9619. doi: $10.1073 /$ pnas.90.20.9616

Sakamoto, K., McCluskey, M., Wensel, T. G., Naggert, J. K., and Nishina, P. M. (2009). New mouse models for recessive retinitis pigmentosa caused by mutations in the Pde6a gene. Hum. Mol. Genet. 18, 178-192. doi: $10.1093 / \mathrm{hmg} / \mathrm{ddn} 327$

Sieving, P. A., Frishman, L. J., and Steinberg, R. H. (1986). Scotopic threshold response of proximal retina in cat. J. Neurophysiol. 56, 1049-1061.
Simonelli, F., Maguire, A. M., Testa, F., Pierce, E. A., Mingozzi, F., Bennicelli, J. L., et al. (2010). Gene therapy for Leber's congenital amaurosis is safe and effective through 1.5 years after vector administration. Mol. Ther. 18, 643-650. doi: $10.1038 / \mathrm{mt} .2009 .277$

Sothilingam, V., Garcia Garrido, M., Jiao, K., Buena-Atienza, E., Sahaboglu, A., Trifunovic, D., et al. (2015). Retinitis pigmentosa: impact of different Pde6a point mutations on the disease phenotype. Hum. Mol. Genet. 24, 5486-5499. doi: $10.1093 / \mathrm{hmg} / \mathrm{ddv} 275$

Strettoi, E., and Pignatelli, V. (2000). Modifications of retinal neurons in a mouse model of retinitis pigmentosa. Proc. Natl. Acad. Sci. U.S.A. 97, 11020-11025. doi: 10.1073/pnas.190291097

Suber, M. L., Pittler, S. J., Quin, N., Wright, G. C., Holcombe, N., Lee, R. H., et al. (1993). Irish setter dogs affected with rod-cone dysplasia contain a nonsense mutation in the rod cGMP phosphodiesterase $\beta$-subunit gene. Proc. Natl. Acad. Sci. U.S.A. 90, 3968-3972. doi: 10.1073/pnas.90.9.3968

Sun, X., Pawlyk, B., Xu, X., Liu, X., Bulgakov, O. V., Adamian, M., et al. (2010). Gene therapy with a promoter targeting both rods and cones rescues retinal degeneration caused by AIPL1 mutations. Gene Ther. 17, 117-131. doi: $10.1038 /$ gt.2009.104

Takahashi, M., Miyoshi, H., Verma, I. M., and Gage, F. H. (1999). Rescue from photoreceptor degeneration in the rd mouse by human immunodeficiency virus vector-mediated gene transfer. J. Virol. 73, 7812-7816.

Tosi, J., Sancho-Pelluz, J., Davis, R. J., Hsu, C. W., Wolpert, K. V., Sengillo, J. D., et al. (2011). Lentivirus-mediated expression of cDNA and shRNA slows degeneration in retinitis pigmentosa. Exp. Biol. Med. 236, 1211-1217. doi: 10.1258/ebm.2011.011053

Tuntivanich, N., Pittler, S. J., Fischer, A. J., Omar, G., Kiupel, M., Weber, A., et al. (2009). Characterization of a canine model of autosomal recessive retinitis pigmentosa due to a PDE6A mutation. Invest. Ophthalmol. Vis. Sci. 50, 801-813. doi: 10.1167/iovs.08-2562

Wensel, T. G., Zhang, Z., Anastassov, I. A., Gilliam, J. C., He, F., Schmid, M. F., et al. (2016). Structural and molecular bases of rod photoreceptor morphogenesis and disease. Prog. Retin. Eye Res. 55, 32-51. doi: $10.1016 /$ j.preteyeres.2016.06.002

Wert, K. J., Davis, R. J., Sancho-Pelluz, J., Nishina, P. M., and Tsang, S. H. (2013). Gene therapy provides long-term visual function in a pre-clinical model of retinitis pigmentosa. Hum. Mol. Genet. 22, 558-567. doi: 10.1093/hmg/dds466

Wert, K. J., Sancho-Pelluz, J., and Tsang, S. H. (2014). Mid-stage intervention achieves similar efficacy as conventional early-stage treatment using gene therapy in a pre-clinical model of retinitis pigmentosa. Hum. Mol. Genet. 23, 514-523. doi: 10.1093/hmg/ddt452

Zhong, L., Li, B., Mah, C. S., Govindasamy, L., Agbandje-McKenna, M., Cooper, M., et al. (2008). Next generation of adeno-associated virus 2 vectors: point mutations in tyrosines lead to high-efficiency transduction at lower doses. Proc. Natl. Acad. Sci. U.S.A. 105, 7827-7832. doi: 10.1073/pnas.0802866105

Zolotukhin, S., Potter, M., Zolotukhin, I., Sakai, Y., Loiler, S., Fraites, T. J. Jr., et al. (2002). Production and purification of serotype 1, 2, and 5 recombinant adeno-associated viral vectors. Methods 28, 158-167. doi: 10.1016/S1046-2023(02)00220-7

Conflict of Interest Statement: WH and the University of Florida have a financial interest in the use of AAV therapies, and own equity in a company (AGTC) that might, in the future, commercialize some aspects of this work.

The other authors declare that the research was conducted in the absence of any commercial or financial relationships that could be construed as a potential conflict of interest.

Copyright (C) 2017 Mowat, Occelli, Bartoe, Gervais, Bruewer, Querubin, Dinculescu, Boye, Hauswirth and Petersen-Jones. This is an open-access article distributed under the terms of the Creative Commons Attribution License (CC BY). The use, distribution or reproduction in other forums is permitted, provided the original author(s) or licensor are credited and that the original publication in this journal is cited, in accordance with accepted academic practice. No use, distribution or reproduction is permitted which does not comply with these terms. 